\title{
Perceptual completion in rhesus monkeys (Macaca mulatta) and pigeons (Columba livia)
}

\author{
KAZUO FUJITA \\ Kyoto University, Kyoto, Japan
}

\begin{abstract}
In a two-dimensional drawing, when the narrow edge of a bar appears to touch the edge of a large rectangle, humans overestimate the length of the bar (Kanizsa, 1979). Kanizsa has suggested that this illusion occurs because humans perceive the bar as continuing behind the rectangle and complete the "occluded" portion of the bar. Rhesus monkeys and pigeons were trained to classify black target bars with a variety of lengths as "long" or "short." In training, the bar was always located at the same distance from a gray box. After learning this discrimination, the subjects were tested on novel stimuli, in which the bar was located at three new locations. Monkeys showed a consistent response bias for "long" when the bar touched the box, but pigeons did not. Monkeys appear to have completed the "occluded" part like humans, whereas pigeons failed to do so. Because this procedure does not require animals to complete the "occluded" part with any particular form, their failure suggests that pigeons do not even perceive the target bar as continuing behind the "occluding" figure. The failure of pigeons may be due to difficulty in perceiving depth from two-dimensional drawings.
\end{abstract}

One of the most important tasks for animals is to identify physical and social objects, such as foods, predators, and companions. In natural environments, however, such objects are often partly hidden by other objects. This means that animals must identify the objects from partial characteristics.

There are two ways of recognizing objects from partial characteristics. One is to establish appropriate responses to each characteristic; for example, antelopes may avoid predation if they take evasive action upon seeing only parts of a lion, such as a mane, a tail, or legs. The other way is to reconstruct a lion from its parts. Which recognition process is used probably depends on context and the lifestyle, genetics, and so forth of the species; hence, this issue is important for understanding adaptive characteristics of species and the evolution of cognition.

Humans recognize the pattern shown in Figure 1 as a triangle on a complete circle, although it could just as well be a triangle touching an incomplete circle. We rou-

This study was supported by Grants-in-Aid for Scientific Research, Ministry of Education, Science, Sports, and Culture, Japan, Nos. 08610082 and 10610072, and by the Cooperation Research Program of Primate Research Institute, Kyoto University. I thank Tetsuro Matsuzawa and So Kanazawa, Primate Research Institute, Kyoto University, for their help in testing monkey subjects. I also thank Sumiharu Nagumo at the same institute for his technical assistance. Thanks are also due James Anderson, University of Stirling, for his editing of an earlier version of the manuscript. Finally, I thank Bruce Schneider, Jim Enns, Sara Shettleworth, and Allison Sekuler for their reviews and useful comments. Correspondence concerning this article should be addressed to K. Fujita, Department of Psychology, Graduate School of Letters, Kyoto University, Sakyo, Kyoto 606-8501, Japan (e-mail: kfujita@ ip.media.kyoto-u.ac.jp or fuijita@psy.bun.kyoto-u.ac.jp). tinely tend to restore the whole from parts (see Grossberg \& Mingolla, 1985; Sekuler \& Palmer, 1992; Yin, Kellman, $\&$ Shipley, 1997). Some forms of such perceptual completion develop early in infancy. For instance, Kellman and Spelke (1983) demonstrated that 4-month-old human infants perceived two rods moving at the same speed and direction behind a screen that hid the central portion as a unitary rod. In adults and older infants, such perception of object unity occurs in the absence of common motion, when two static rods are merely aligned to make good continuity.

Sato, Kanazawa, and Fujita (1997) recently demonstrated that an adult chimpanzee showed similar perception. They trained the subject on a matching-to-sample of one rod and two rods. In probe test trials, the chimpanzee matched two rods moving in concert with the central portion occluded, with one rod. On the other hand, she matched two rods moving out of concert with two rods. Subsequent tests revealed that the chimpanzee also perceived object unity when two static rods were well aligned. Thus, the chimpanzee's perception of object unity was more like that of adult humans than that of young human infants.

Kanizsa, Renzi, Conte, Compostela, and Guerani (1993) reported that mice showed responses consistent with perceptual completion after being trained to discriminate complete figures and incomplete ones, although the authors noted other possible explanations for the animals' responses as well.

On the other hand, pigeons are consistently reported not to complete the "hidden" parts of a figure. Cerella (1980) trained pigeons to discriminate a complete triangle from other figures. In subsequent tests, the pigeons consistently reported incomplete triangles touching a box (as if the lat- 


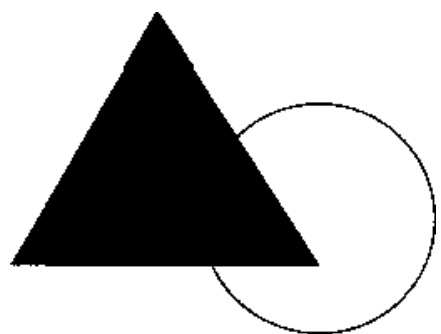

Figure 1. An example of perceptual completion.

ter was hiding the former) as "no triangle." More recently, Sekuler, Lee, and Shettleworth (1996) also reported that pigeons completed neither a circle nor a rectangle.

The above studies show that pigeons do not perceive the "completed" object as humans do. For example, pigeons do not seem to perceive a circle in Figure 1. There are several possible reasons why pigeons fail to complete occluded objects as humans do. One possibility is that pigeons indeed perceive a figure behind the triangle but fail to identify its shape. A second possibility is that pigeons perceive some figure that is different from what we perceive; the Gestalt principle of good shape may not be shared by different species. A third possibility is that they do not perceive the triangle in Figure 1 to be blocking or occluding another figure. In other words, pigeons may not even perceive continuation of the "occluded" figure behind the "occluder."

In the present study, we test this third, most extreme hypothesis. To do so, we investigate a perceptual illusion, described below, that presumably arises when a figure is seen as continuing behind an occluding shape in a twodimensional (2-D) scene but that does not depend on the identification of the occluded object. If pigeons fail to perceive the illusion, we may safely conclude that pigeons do not perceive continuation of the figure behind the occluder.

Kanizsa (1979) showed that humans overestimate the length/size of a smaller figure touching a larger figure. For example, in the top of Figure 2, the trapezoid touching the large rectangle looks larger than the other three, and in the bottom of the same figure, the lower dark rectangle looks longer than the one above. According to Kanizsa, this size overestimation occurs because we perceive that the large rectangle "hides" the end of the smaller figures touching it, and hence we complete the "hidden" part of the smaller figures.

This is probably one of the simplest cases in which perceptual completion could occur. All that animals have to do to perceive these illusions is to recognize that the "occluded" figure simply continues behind the "occluder." These illusions should occur no matter what the "good form" is for the particular animals, because they are not required to identify the "occluded" figure. If pigeons, when presented with a 2-D drawing, such as those in Figure 1 or 2 , see one shape as continuing behind another (even though they cannot identify the occluded shape), they should also be susceptible to the illusion.

The purpose of the present experiments was to reexamine perceptual completion in this context for pigeons and to compare perceptual completion in pigeons with that of nonhuman primates. In Experiment 1, rhesus monkeys were tested. Pigeons were tested in the three subsequent experiments.

\section{EXPERIMENT 1}

\section{Method}

Subjects. Two rhesus monkeys (Macaca mulatta) kept at the Primate Research Institute, Kyoto University, were used. One was a 10 -year-old male (Gonta), and the other was a 9-year-old female (Jusco). They were not deprived of food but were fed after the experimental session, once each day. Both subjects had experience of simple leverpressing for visual reinforcers and of visual discrimination tasks using a touch panel, but neither had been trained to complete or to perceive three-dimensional (3-D) relations from 2-D drawings.

Apparatus. An experimental box $(70 \times 70 \times 70 \mathrm{~cm})$ was located in a dimly lit room. One wall of the box had an opening $(40 \mathrm{~cm}$ wide $\times 30 \mathrm{~cm}$ high). A 14-in. monitor (Sony, KX-14HD) with a static-capacitance touch sensor (Nissha Intersystems, Hypertouch) installed was placed immediately behind the opening. A universal feeder (Sanso, S-100) was positioned on the top of the box to deliver food into a cup on the side wall. A computer (Epson, PC-286VE) controlled the apparatus and collected the data.

Stimuli. Figure 3 shows examples of the stimuli. Black computer graphics on a white background were used. The spatial resolution was $640 \times 400$ pixels. The target stimulus was a black horizontal bar presented at the center of the monitor. The thickness of the bar was 15 pixels, and the length varied in 3-pixel steps from 45 to
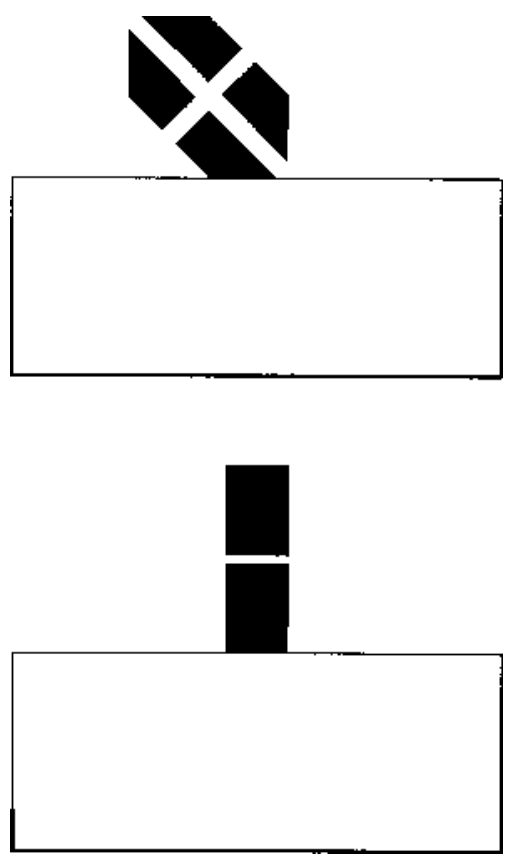

Figure 2. Figures redrawn from Kanizsa (1979). 

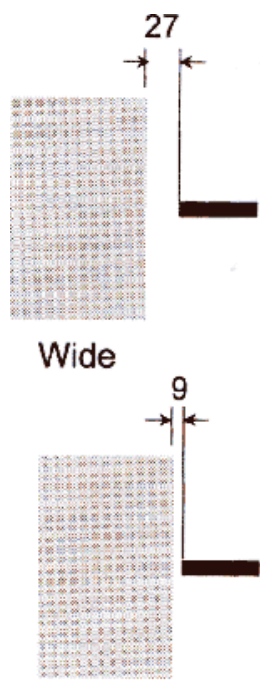

Narrow

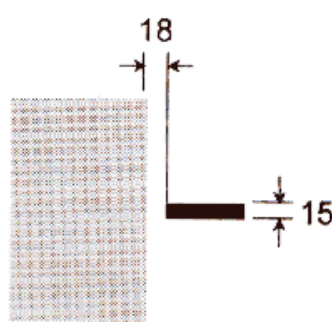

Baseline

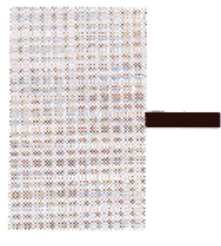

Touch
Figure 3. Examples of the stimuli used in Experiment 1. The numbers in the figure are in graphic pixels.

90 pixels during training (i.e., 16 different lengths). There was a gray box $(120 \times 195$ pixels $)$ either to the right or to the left of the target bar. The size of the gap between the gray box and the target bar was 18 pixels in training and $27,18,9$, or 0 pixels in testing. These were labeled wide, baseline, narrow, and touch conditions, respectively. On the display, 100 pixels extended $44 \mathrm{~mm}$. The viewing distance was not controlled, but the monkeys typically held to a distance of approximately $15-30 \mathrm{~cm}$. When viewed from this distance, the visual angle of the 45 -pixel bar was $7.57^{\circ}-3.78^{\circ}$, and that of the 90-pixel bar was $15.13^{\circ}-7.57^{\circ}$.

Procedure. Figure 4 shows a schematic representation of the task. The task was to classify the target bar as "long" or "short." A stimulus pattern appeared after an intertrial interval of $3 \mathrm{sec}$, during which the display was white. When the subject touched the target bar five times, two choice locations appeared at the bottom two corners of the display. The one on the right was a solid rectangle, and the one on the left was an open rectangle; both were 60 pixels wide and 40 pixels high. One of 16 different lengths of the target bar appeared randomly on each trial. Responses on one of the two choice locations were correct for the eight long bars, whereas responses on the other were correct for the eight short bars (i.e., a conditional position discrimination task). The relationship between the length of the bar and the correct choice location was counterbalanced across the subjects. On half of the trials, correct responses were reinforced by a piece of sweet potato or monkey chow, accompanied by a 1 -sec electronic doorbell sound. On the remaining half of the trials, correct responses were conditionally reinforced by the same doorbell sound of $0.5 \mathrm{sec}$. That is, the probability of primary reinforcement was .5. Incorrect responses were followed by a 5 -sec timeout, during which the display was completely dark.

Since both subjects had learned a similar task before the start of this experiment (Fujita, 1996, 1997), they started on the baseline discrimination with the isolated target bar (i.e., without the gray box). Sessions consisted of 192 trials. After the subjects scored higher than $85 \%$ correct for two consecutive sessions, the gray box was introduced at the position 18 pixels distant from the near end of the target bar. The left-right position of the gray box was counterbalanced across trials.
After the subjects scored higher than $85 \%$ correct for two consecutive sessions, they entered a test phase. During test sessions, the length of the target bar was $51,57,63,66,69,72,78$, or 84 pixels. There were 192 trials in each test session, and tests were done with two consecutive sessions as a block. Of the 384 trials of each block, half were baseline (training) trials, in which choice responses were differentially reinforced, and the others were test trials, in which choice responses were nondifferentially reinforced at the same probability of primary reinforcement as the training trials (i.e., .5). Wide, baseline, narrow, or touch conditions appeared randomly at the same frequency among the test trials. Thus, each of the four conditions was tested 48 times in each block. In the 48 trials, each stimulus configuration ( 8 different lengths of the target bar $\times$ 2 locations of the gray box) was featured 3 times. At least one training session separated the test blocks. The test blocks were run 12 times; hence, 36 trials were tested for each stimulus conf iguration.

After the test phase, the subjects were given one session in which no gray box appeared, in order to ensure that discrimination was based solely on the length of the target bar. The contingency of reinforcement was exactly the same as that in the baseline sessions.

\section{Results and Discussion}

Neither monkey showed a deterioration in performance when tested with isolated target bars after the test period, confirming that their discrimination was based on the length of the target bar.

Figure 5 shows the proportion of choice responses on the "long" key as a function of the length of the target bar. The top two graphs are for each subject, and the bottom graph shows the average. The data for the training trials and the baseline test trials during test sessions were summed, because there were no clues for the subjects to discriminate these two types of trials before making a choice response.

A two-way analysis of variance (ANOVA) of stimulus condition $\times$ bar length conducted on the averaged data, with subjects treated as a randomized block, indicated significant main effects of stimulus condition $[F(3,31)=$
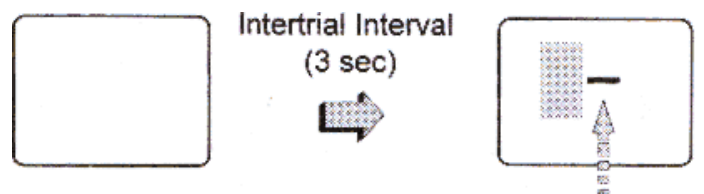

5 Responses

\section{Reinforcement}
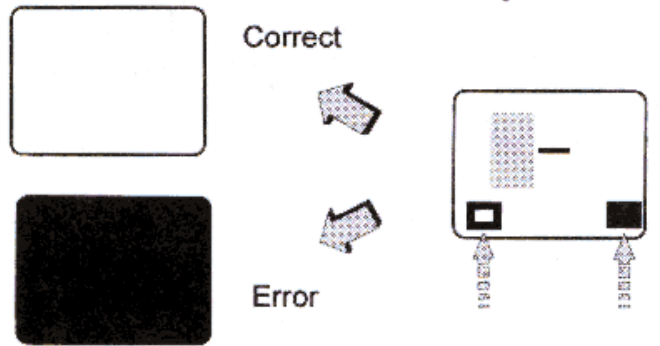

Timeout
Correct
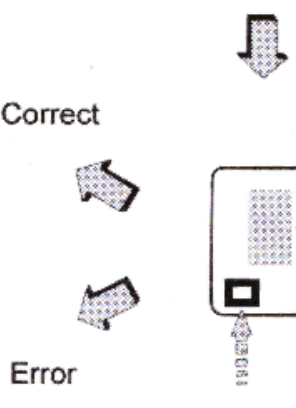

Figure 4. A schematic drawing of the task. 

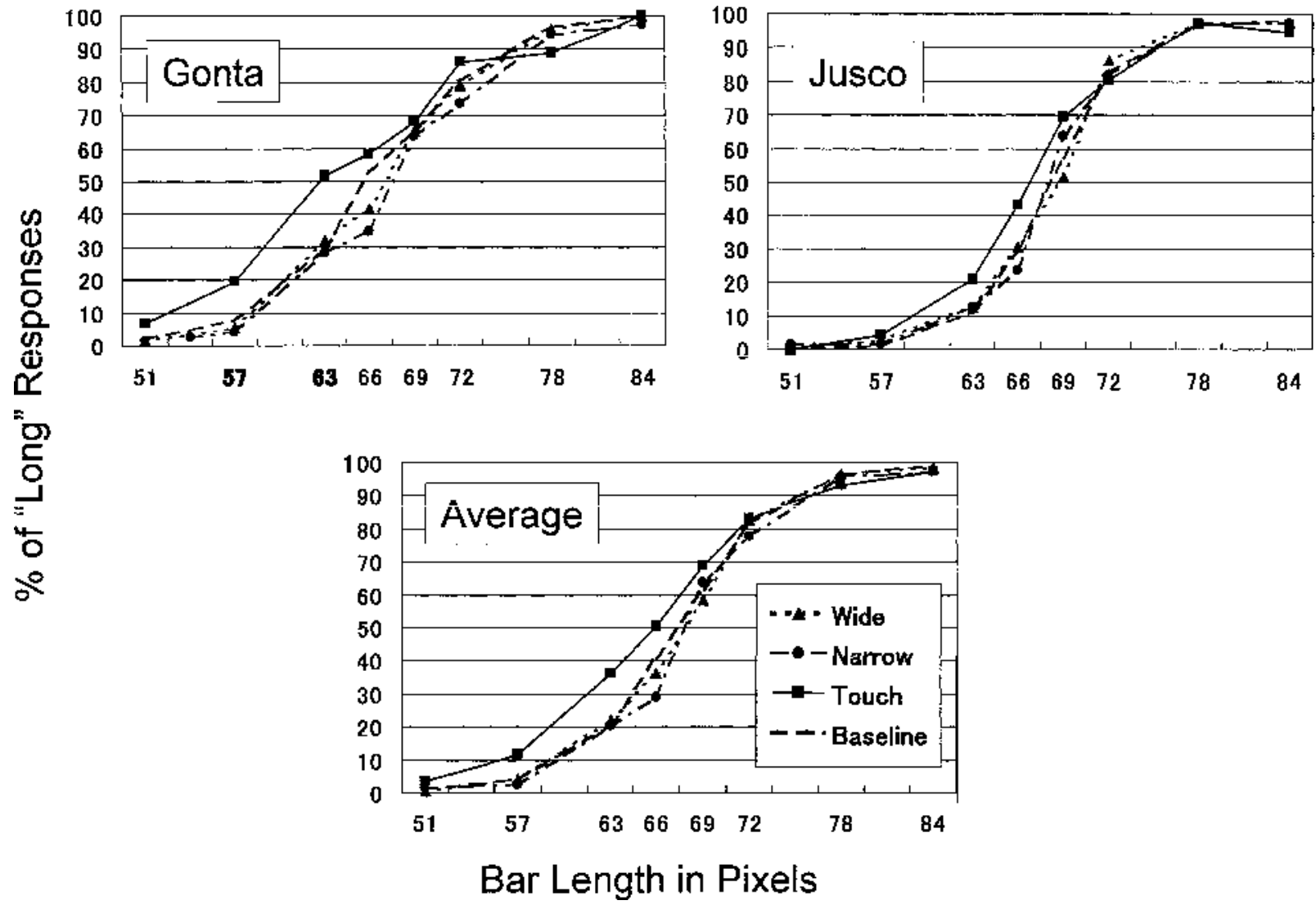

Figure 5. The results of Experiment 1 with monkeys. The top two graphs are for individual monkeys, and the bottom one is the average. The vertical axis is the proportion of choice responses on the long key, and the horizontal axis is the length of the target bar in graphic pixels. The dashed line with no symbols is the sum of the training trials and the baseline test trial; other lines with symbols are the other test trials.

3.43, $p=.029]$ and bar length $[F(7,31)=276.21, p=$ $.000]$. The interaction was not significant $[F(21,31)=$ $0.74, p=.757]$. Because the proportions of "long" responses for all the conditions were close to 0 for the shortest two lengths and close to 100 for the longest two, the same ANOVA was conducted for only the middle four points (i.e., 63, 66, 69, and 72). The results of this second analysis were similar to the first $[F(3,15)=3.55, p=.040$; $F(3,15)=80.26, p=.000 ; F(9,15)=0.59, p=.786$, respectively]. For the purpose of post hoc comparison among the stimulus conditions, a third ANOVA, with the subjects treated as random error, was conducted. The main effect of stimulus condition only approached significance in this case $[F(3,32)=2.51, p=.077]$. This was clearly because the main effect of block (subject) in the first ANOVA was highly significant $[F(1,32)=12.72, p=$ $.001]$, owing to the monkeys' response bias on the choice locations. However, a post hoc Tukey least significant difference (LSD) analy sis revealed the touch condition to be significantly different from the wide and the narrow conditions ( $p=.046$ and $p=.015$, respectively), whereas the difference from the baseline condition approached significance $(p=.084)$.
Therefore, the subjects consistently tended to choose "long" more often in the touch condition than the other three conditions - that is, they overestimated the length of the bar touching a larger box. This tendency was observed in both the subjects. The magnitude of this overestimation for the touch condition, as compared with the baseline condition, was $3.1 \%$ at the point at which the choice response was $50 \%$. A plausible interpretation of this tendency is that the monkeys perceived continuation of the target bar behind the gray box, thus completing the "occluded" part of the target bar in some manner.

Alternatively, the overestimation in the touch condition could conceivably be a consequence of assimilation to and the contrast with the gray rectangular box. As long as there are two or more figures in a display, there is always room for such interaction among the figures, and the way they interact depends on the distance between the figures. For example, in the Delboeuf illusion, the inner one of two concentric circles assimilates to the outer one to look larger than it actually is when the difference in size of the two circles is small. However, as the outer circle becomes larger, the inner one starts to shrink by virtue of a contrast effect with the larger one. If this were the case 
in the present experiment, however, the magnitude of the overestimation (or underestimation) should change systematically as a function of the size of the gap between the bar and the rectangle. As can be seen in Figure 5, however, there was very little difference in the proportion of "long" choices among the wide, narrow, and baseline conditions having different gap sizes. Therefore, it is unlikely that the observed overestimation in the touch condition was due to such perceptual processes, although there remains the slight possibility that such effects appear only for distances in between those in the touch and the narrow conditions.

In summary, the results from this experiment suggest (1) that the procedure used allows us to analyze perceptual completion processes of nonhuman animals based on the perception of mere continuation, regardless of what may be a "good shape," and (2) that rhesus monkeys appear to perceive continuation of the bar behind the box (a form of completion of the "occluded" part of the figure touching a larger "overshadowing" figure).

\section{EXPERIMENT 2}

In this experiment, pigeons were tested for an illusion similar to that shown with rhesus monkeys in Experiment 1 , using exactly the same procedure.

\section{Method}

Subjects. Three 7-month-old experimentally naive homing pigeons (Columba livia) kept at the Faculty of Letters, Kyoto University, were used. Two were males (Pigeons 5 and 6) and one was a female (Pigeon 7). Their body weights were maintained at approximately $90 \%$ of their free-feeding weights throughout the experimental period.

Apparatus. Three experimental boxes $(40 \times 40 \times 40 \mathrm{~cm})$ were used. One wall of the box contained an opening $(20 \mathrm{~cm}$ wide $\times$ $18 \mathrm{~cm}$ high), behind which a 15-in. monitor (Sony, CPD15-SF8) with an infrared touch sensor (Carrol Touch, Unitouch) was placed. A grain feeder was attached to the side wall of the box. A computer (Fujitsu, FM/V-133SP or FM/V-133SE) controlled the apparatus and collected the data.

Stimuli. Black computer graphics on a white background were used. The spatial resolution was $640 \times 480$ pixels. The sizes of all stimuli were one third of those used for the monkeys- that is, the thickness of the target bar was 5 pixels, and the length varied in 1pixel steps from 15 to 30 pixels (i.e., 16 different lengths). This was done because pigeons typically observe the stimuli at a distance of $5-10 \mathrm{~cm}$. The gray box was $40 \times 65$ pixels, either to the right or to the left of the target bar. The size of the gap between the gray box and the target bar was 6 pixels in training and 9, 6, 3, or 0 pixels in testing (wide, baseline, narrow, and touch conditions, respectively). On the display, 100 pixels extended $43 \mathrm{~mm}$. Viewed from a distance of 5-10 cm, the visual angle of the 15-pixel target bar was $7.39^{\circ}-$ $3.70^{\circ}$ and that of the 30-pixel target was $14.79^{\circ}-7.39^{\circ}$.

Procedure. The pigeons were first shaped to peck at a small black square at the center of the display. Then, they were trained on discrimination of the bar length. The procedure was exactly the same as that in Experiment 1. The relationship between the length of the bar and the correct choice location was counterbalanced across the subjects. The pigeons started with discrimination of bars 5 and 40 pixels in length without the gray box. Next, they were trained on a discrimination of bars of 15 and 30 pixels. Finally, they were trained to discriminate all 16 bar lengths. Initially, all the correct responses were reinforced with 3 -sec access to mixed grain, accompanied by illumination of the feeder. In the later stages, they were reinforced only on one third of the trials. Correct responses not followed by food were secondarily reinforced by a 3-sec illumination of the feeder. Sessions consisted of 384 trials. Incorrect choice responses were followed by a time-out of $5 \mathrm{sec}$.

When the birds scored higher than $75 \%$ for two consecutive sessions, the gray box was added. ${ }^{1}$ The left-right position of the gray box was counterbalanced. After the subjects performed at more than $75 \%$ correct for two consecutive sessions, they entered the test phase. During test sessions, the length of the target bar was 17, 19, $21,22,23,24,26$, or 28 pixels. Each test session consisted of 384 trials. Half of the trials were baseline (training) trials, in which choice responses were differentially reinforced, and the others were test trials, in which choice responses were nondifferentially reinforced at the same probability as baseline trials. Wide, baseline, narrow, or touch conditions appeared randomly at the same frequency among the test trials. Thus, each of the four conditions was tested 48 times in each block. Among the 48 trials, each stimulus configuration ( 8 different lengths of the target bar $\times 2$ locations of the gray box) was tested 3 times. Test sessions were separated by at least one training session. The test was run 12 times; hence, each stimulus configuration was featured in 36 trials.

After the test period, the subjects were given one session in which no gray box appeared, to ensure that their discrimination was based on the target bar.

\section{Results and Discussion}

No birds showed a deterioration in performance when tested with isolated target bars after the test period, indicating that discrimination was based on the length of the target bar.

Figure 6 shows the proportion of choice responses on the "long" key as a function of the length of the target bar. The bottom right graph shows the average for the three birds, and the others graphs are for individual subjects. As in Figure 5, the data for the training trials and the baseline test trials during the test sessions were summed, because there were no clues for the birds to discriminate in these two types of trials before making a choice response.

As in Experiment 1, a two-way ANOVA of stimulus condition $X$ bar length was conducted on the averaged data, with the subject treated as a randomized block. The main effect of stimulus condition $[F(3,62)=3.16, p=.031]$ and the main effect of bar length $[F(7,62)=163.69, p=$ $.000]$ were significant. The interaction was not $[F(21,62)=$ $0.70, p=.819]$. The significant effect of stimulus condition, however, was due to more frequent choices of "long" not in the touch condition, but in the wide condition. This contrasts with the results obtained with monkeys in Experiment 1 .

If the subjects were treated as random error, there was no significant difference in the main effect of stimulus condition $[F(3,64)=0.79, p=.504]$, because of the strong main effect of the block (subject) in the first ANOVA $[F(2,62)=97.19, p=.000]$ owing to the birds' response bias on the choice locations.

In summary, in contrast to the results from Experiment 1 with monkeys, pigeons showed no bias for reporting "long" more often when the target bar touched the 

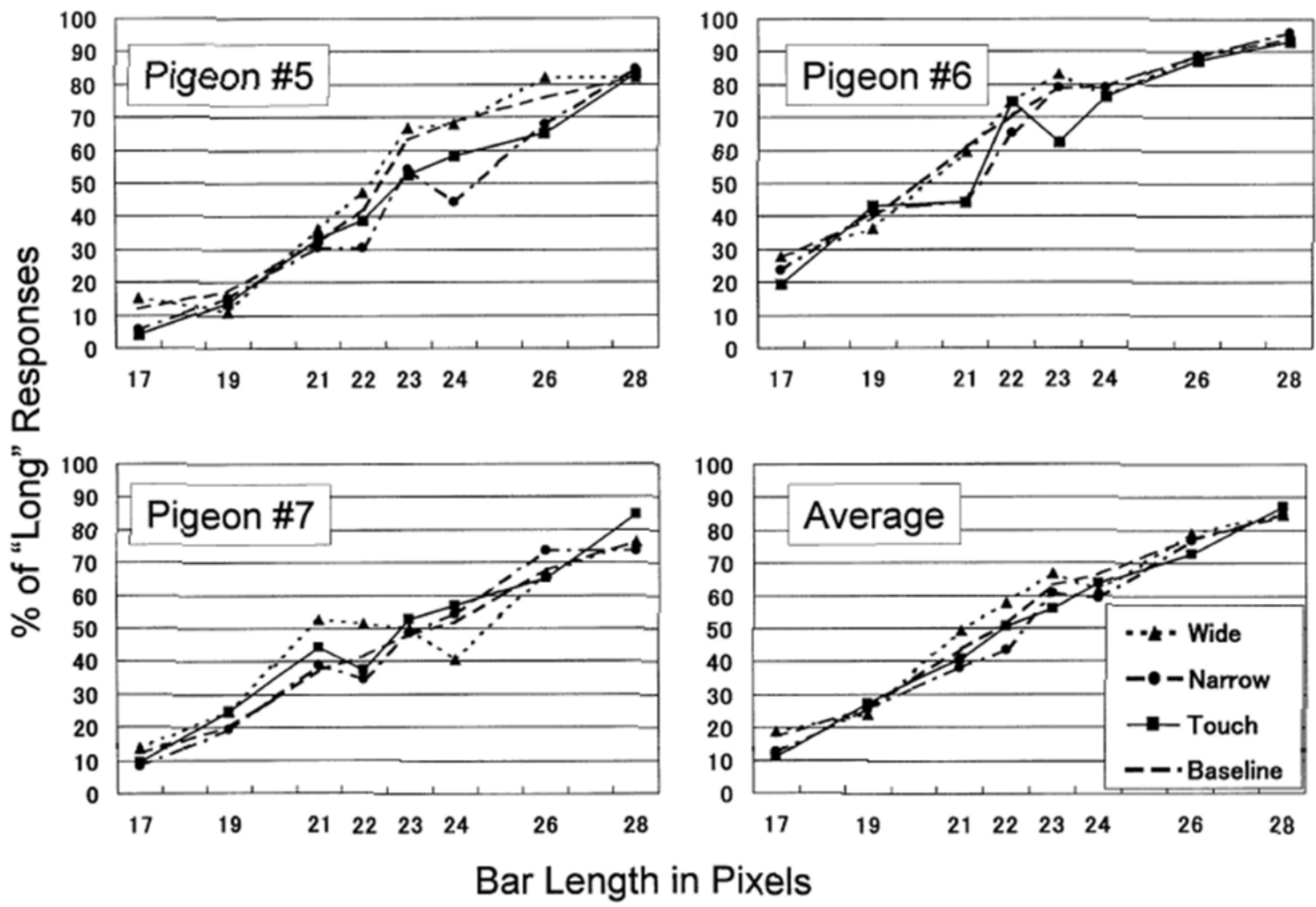

Figure 6. The results of Experiment 2 with pigeons. The graph at the bottom right is the average, and the others are for individual birds. The vertical axis is the proportion of choice responses on the long key, and the horizontal axis is the length of the target bar in graphic pixels. The dashed line with no symbols is the sum of the training trials and the baseline test trial; other lines with symbols are the other test trials.

box. We obtained no evidence that the pigeons continued the "hidden" part of the target bar.

\section{EXPERIMENT 3}

Examination of Figure 6 raises the possibility that the proportion of "long" choices may be higher in the wide condition than in the narrow condition, suggesting a possible contrast effect provided by the large closed box, as was discussed in Experiment 1. If real, this tendency might have masked any effect of perceptual completion. Since we have no knowledge of how such a contrast effect changes as a function of the size of the gap in pigeons in this situation, we cannot yet conclude that pigeons fail to perceptually continue "hidden" parts of figures.

In Experiment 3, a gray area (described below) was used in place of the gray box. We expected that the gray area would be too large for pigeons to perceive as a closed figure; hence, this procedure was expected to minimize the contrast effect discussed above.

\section{Method}

Subjects. Two of the 3 pigeons used in Experiment 1 (Pigeons 6 and 7) were used, and another naive male of the same age (Pigeon 8) replaced Pigeon 5, who was retired because of illness. As in Experiment 2, the pigeons' body weights were maintained at about $90 \%$ of their free-feeding weights. Experiment 3 started immediately after Experiment 2.

Apparatus. The same experimental boxes as those in Experiment 2 were used.

Stimuli. The stimuli were the same as those in Experiment 2, except that a gray area replaced the gray box. For presenting a gray area, half of the monitor screen became homogeneously gray. This gray area appeared either to the right or to the left of the target bar.

Procedure. Pigeon 8 went through the same procedure as that used in Experiment 2 to establish baseline discrimination of the length of the target bar. The other pigeons started with the same baseline discrimination as that in Experiment 1. When the birds scored higher than $75 \%$ for two consecutive sessions, the gray area was introduced, with left-right position of the gray counterbalanced. After the subjects performed at more than $75 \%$ correct for two consecutive sessions, they entered the test phase. The test was conducted in exactly the same manner as that in Experiment 2. After the test phase, the subjects were given one session without the gray area, to ensure that their discrimination was based on the target bar.

\section{Results and Discussion}

No birds showed a deterioration of performance when tested with isolated target bars after the test period. This again confirmed that the pigeons' discrimination was based on the length of the target bar. 

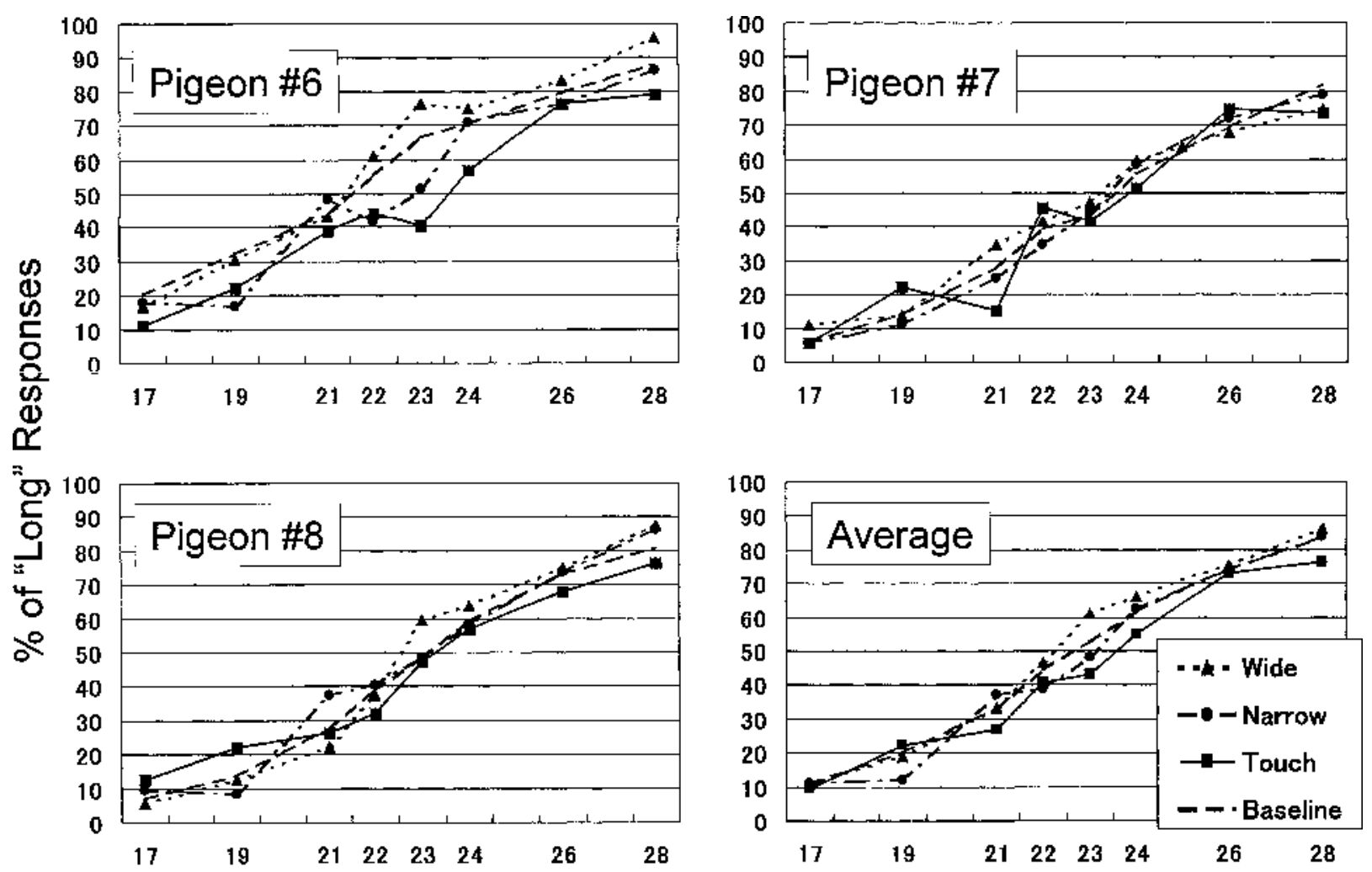

Bar Length in Pixels

Figure 7. The results of Experiment 3 with pigeons. The graph at the bottom right is the average, and the others are for individual birds. The vertical axis is the proportion of choice responses on the long key, and the horizontal axis is the length of the target bar in graphic pixels. The dashed line with no symbols is the sum of the training trials and the baseline test trial; other lines with symbols are the other test trials.

Figure 7 shows the proportion of choice responses on the "long" key as a function of the length of the target bar. The bottom right graph shows the average of the three birds, and the others are for each subject.

As in Experiment 2, the proportion of "long" choices was not higher in the touch condition than in the others. When the same two-way ANOVA of stimulus condition $X$ bar length was conducted for the averaged data, with the subject treated as a randomized block, the main effect of stimulus condition $[F(3,62)=6.91, p=.000]$ and the main effect of bar length $[F(7,62)=301.82, p=.000]$ were significant, whereas the interaction was not $[F(21,62)=$ $1.47, p=.122]$. The main effect of stimulus condition was significant even when the subjects were treated as random error $[F(3,64)=2.87, p=.043]$. Figure 7 shows that the significant effect of stimulus condition appears to have resulted from the higher proportion of "long" choices in the wide condition and the lower proportion in the narrow and the touch conditions. A post hoc Tukey LSD analysis indicated a significant difference between the wide and the touch conditions $(p=.006)$ and a trend between the wide and baseline conditions and between the narrow and the wide conditions.

Consequently, the difference in performance across the four conditions appeared larger in this experiment than in Experiment 2. This difference is interesting, and why such illusory effects should be stronger with the gray area, rather than with the closed box, should be addressed in the future. But in the context of the present study, the most important finding was again the absence of any evidence that pigeons continued the "hidden" part of the target bar behind the occluding region.

\section{EXPERIMENT 4}

We failed to obtain evidence for perceptual continuation by pigeons in the preceding two experiments. However, the magnitude of overestimation found in monkey subjects in this experimental situation was only about $3 \%$. Discrimination accuracy in the baseline trials during test sessions was much lower in pigeons (mean, $69.2 \%$ in Experiment 2 and $70.4 \%$ in Experiment 3 ) than in mon- 


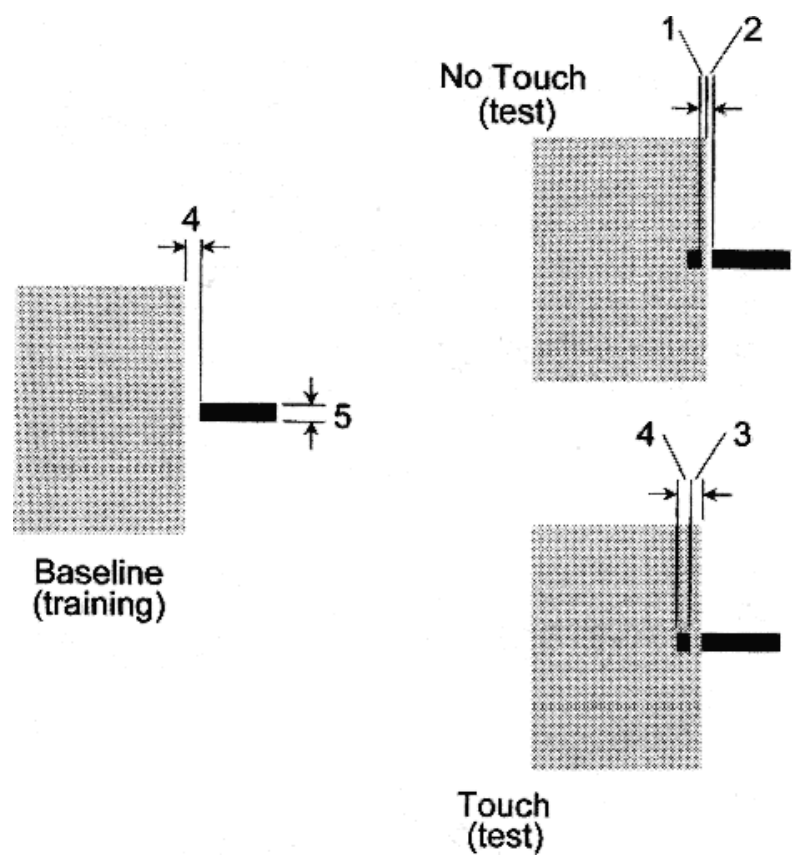

Figure 8. Examples of stimuli used in Experiment 4. The numbers in the figure are in graphic pixels.

keys (mean, $84.1 \%$ ), and this relatively poor discrimination performance might have obscured any subtle effect in pigeons.

In Experiment 4, pigeons were tested in a situation in which a stronger reporting bias might be expected.

\section{Method}

Subjects. The same 3 pigeons as those used in Experiment 3 were used. Their body weights were maintained at about $90 \%$ of their free-feeding weights. Experiment 4 started immediately after Experiment 3.

Apparatus. The same experimental boxes as those used in Experiment 2 were used.

Stimuli. Figure 8 shows examples of the stimuli. The training stimuli were very similar to those used in Experiment 3, but the gap between the target bar and the gray area was 4 pixels, rather than 6 pixels, as in Experiments 2 and 3. There were two types of test stimuli. One was the no-touch condition, in which a target bar was 2 pixels apart from the gray box and a small black rectangle, 3 pixels wide and 5 pixels high, was placed 1 pixel inside the edge of the gray area. The other was the touch condition, in which a target bar touched the gray area and the same small black rectangle was placed 3 pixels inside the edge of the gray area. To human eyes, the black bar appears to slice through the background to be a unitary black bar (object unity) only in the touch condition; hence, it appears to be much longer than in the other conditions. The gray area appeared either to the right or to the left of the target bar.

Procedure. All the pigeons had mastered the baseline discrimination without the gray area when this experiment started. When the birds scored higher than $75 \%$ correct for two consecutive sessions, the gray area was added in counterbalanced left-right position. After the subjects had performed at higher than $75 \%$ correct for two consecutive sessions, they entered into the test phase. The test was conducted in a manner similar to that in Experiments 2 and 3. Test sessions consisted of 384 trials, of which two thirds were baseline trials and the remaining one third were test trials in which all choice responses, either long or short, were nondifferentially reinforced. The probability of the primary reinforcement was the same as that in Experiments 2 and 3 for both the baseline and the test trials. The stimuli for the no-touch and the touch conditions appeared randomly at the same frequency among these test trials. The test was run 12 times, so that 48 trials for each stimulus configuration were presented in total. After the test period, the subjects were given one session without the gray area, to ensure that their discrimination was based on the target bar.

\section{Results and Discussion}

Again, no birds showed deterioration of performance when tested with isolated target bars after the test period.

Figure 9 shows the proportion of choice responses on the "long" key as a function of the length of the target bar in pixels. The bottom right graph is for the average of the 3 birds, and the others are for each subject.

The proportion of "long" choices was consistently higher in the touch and the no-touch conditions than in the baseline condition. When the same two-way ANOVA of stimulus condition $\times$ bar length was conducted for the averaged data, with the subject treated as a randomized block, the main effect of stimulus condition $[F(2,46)=42.32$, $p=.000]$ and the main effect of bar length $[F(7,46)=$ $56.95, p=.000]$ were highly significant. The interaction was not significant $[F(14,46)=0.75, p=.715]$. The main effect of stimulus condition was significant even when the subjects were treated as random error $[F(2,48)=9.51, p=$ $.000]$. A post hoc Tukey LSD analysis suggested a highly significant difference between the touch and the baseline conditions and between the no-touch and the baseline conditions ( $p=.000$ for both). There was no difference between the touch and the no-touch conditions $(p=.836)$. It seems likely that the pigeons reported the total length of the target bar plus the small rectangle, regardless of whether there was a gap between the bar and the gray area or not.

It is theoretically possible that the pigeons were unable to detect the gap of 2 pixels between the target bar and the gray area in the no-touch condition. However, this is unlikely, given that the visual acuity of pigeons is 12.7 cycles/deg or higher (Hodos, 1993) and a single pixel in this display subtended an angle between $0.49^{\circ}$ and $0.25^{\circ}$ when viewed from a distance of between 5 and $10 \mathrm{~cm}$. Thus, the lack of any difference in the proportion of choice for "long" between the touch and the no-touch conditions again suggests failure of the pigeons to complete the occluded portions of the target bar.

\section{GENERAL DISCUSSION}

Experiment 1 demonstrated that rhesus monkeys overestimated the length of a target bar by about 3\% when it touched a larger box. According to Kanizsa (1979), humans overestimate the target shown in Figure 2 by about $8 \%$. Kanizsa's procedure involves simultaneously comparing the sizes of the two figures, a situation that should produce a more consistent and somewhat stronger illusion than successively comparing the sizes of the two 

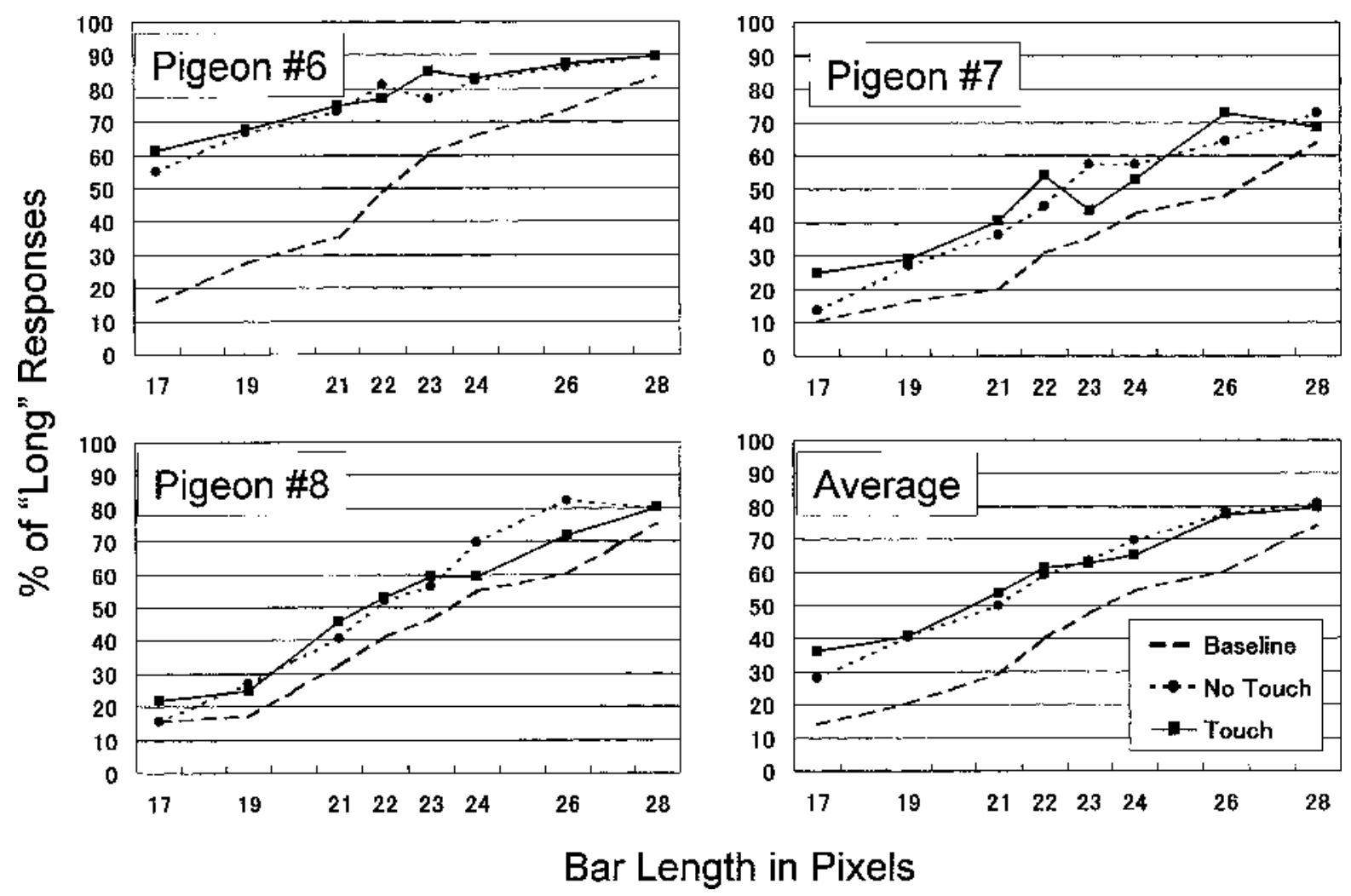

Figure 9. The results of Experiment 4 with pigeons. The graph at the bottom right is the average, and the others are for individual birds. The vertical axis is the proportion of choice responses on the long key, and the horizontal axis is the length of the target bar in graphic pixels. The dashed line with no symbols is the sum of the training trials and the baseline test trial; other lines with symbols are the other test trials.

figures. If we take procedural difference into account, the magnitude of the monkeys' illusion in the present experiment does not appear very different from that of humans. Processes of perceptual continuation by humans and monkeys may be similar both qualitatively and quantitatively.

In Experiments 2-4, a similar illusion was examined in pigeons. Experiment 2, in which exactly the same procedure as that in Experiment 1 was used, failed to demonstrate perceptual continuation in pigeons. In Experiment 3 , in which the potential effect of contrast from the large gray box was expected to be minimal, we again obtained no evidence for perceptual continuation by pigeons. However, pigeons tended to overestimate bars that were more distant from the gray area. This illusion merits further investigation. In Experiment 4, in which the response bias to show completion was expected to be larger than in Experiments 2 and 3, there was no evidence that pigeons completed the hidden portion. These negative results are consistent with previous studies, suggesting that pigeons do not complete partly occluded objects (Cerella, 1980; Sekuler et al., 1996). In addition, the present results provide strong evidence that pigeons may not even perceive continuation of the figure behind the occluder.
Interestingly, domestic chicks tested using imprinting procedures have been suggested to perceive object unity under conditions of partial occlusion(Lea, Slater, \& Ryan, 1996; Regolin \& Vallortigara, 1995). Although their procedures are considerably different from those used in pigeon studies, such a result suggests that the ability for completion does not seem to be beyond the neural systems of birds. But the visual capability of pigeons may be different from that of other birds in an important way; pigeons may not even recognize food, or at least they lose interest in it, when it becomes invisible behind a screen, whereas mynahs maintain their interest (Plowright, Reid, $\&$ Kilian, 1998). Poor recognition of hidden objects was also reported in a species closely related to pigeons (ring doves; Dumas \& Wilkie, 1995). On the other hand, psittacine birds, such as parrots and parakeets, have been demonstrated to show good object permanence (Funk, 1996; Pepperberg \& Funk, 1990). The difference in the ability to find hidden objects between pigeons and these other birds might be partially understandable from their feeding habits. Pigeons are grain eaters. Grains are abundant, and there may be little need for pigeons to search for grains behind obstacles. On the other hand, other birds feed on more varied foods. Domestic chicks and mynahs eat worms as 
well as grains. Worms often hide under leaves or soil, becoming only partially visible. Predators ought to recognize partially occluded prey to enable them to search for prey behind obstacles. Also, larger species of parrots and parakeets sometimes crack open hard nuts to eat the kernel. Such behavior also requires the ability to recognize hidden edible parts. It is possible that the ability to recognize hidden objects is better developed in predators and extractive foragers, and this in turn might be related to the ability for visual completion. A test of this hypothesis requires a large-scale cross-species study.

The present results suggest a marked difference between primates and pigeons in the potential to recognize continuation of a partially "occluded" figure behind the "occluder" and to complete "hidden" portions in 2-D figures. However, before we conclude that pigeons always fail in such tasks, we have to address at least the following three points.

First, there is the possibility that the procedure used in this study was not appropriate for pigeons. This is unlikely because, in our previous studies on the Ponzo illusion, using a similar procedure, pigeons did show a bias of reporting "long" and "short" (Fujita, Blough, \& Blough, 1991, 1993).

Second, perhaps pigeons failed to recognize 3-D in the 2-D figures. In order for continuation or completion to occur, the depth relationship must be recognized. When humans perceive a complete circle in Figure 1, they perceive the triangle as being closer to them than the "circle." Pigeons are known to have difficulty recognizing depth from 2-D drawings (e.g., Blough, 1984; Cerella, 1990), unless they are specially trained to recognize rotating 2-D representations of figures (Cook \& Katz, 1999). In contrast, humans start to recognize 3-D when they are only a few months old (see, e.g., Granrud \& Yonas, 1984; Yonas \& Arterberry, 1994), as may macaque monkeys (Gunderson, Yonas, Sargent, \& Grant-Webster, 1993) and domestic hens (Forkman, 1998). If the pigeons in this experiment did not perceive 3-D in the stimuli presented, it means that this experimental situation did not provide them with a condition necessary for them to perform perceptual completion. It remains an open question whether pigeons can complete real partly occluded 3-D objects. However, regardless of the outcome of such tests, there remains a clear interspecies difference in the ability to complete partly occluded 2-D objects; pigeons seem not to have this ability, whereas all other species tested do.

The third point is that there are clues for completing hidden parts other than occlusion. For instance, as was described in the introduction, human infants start to perceive object unity earlier when the two parts move in concert than when they are static. That is, in humans, completion cued by unison of motion comes earlier in the ontogenetic process than does completion cued by good alignment. If a similar temporal relation is replicated in phylogenetic terms, pigeons may complete only stimuli showing common motion. This possibility requires investigation.

In summary, in this study, perceptual completion in rhesus monkeys and pigeons was examined. Whereas monkeys showed evidence of completion, pigeons did not. The failure of pigeons is not only consistent with previous reports but also demonstrates that pigeons may not even recognize continuation of partly occluded figures behind an occluder. We may be able to trace the phylogeny of perceptual completion by testing a variety of different species, using similar procedures. Studies of perceptual completion help to understand the cognitive characteristics of species. Animals have perfect perceptual freedom regarding how they complete hidden parts. In such situations, the psychological characteristics of species ought to reveal themselves clearly. In this sense, studies of perceptual completion may be called a perceptual projection method, like the Rorschach or TAT techniques, and could have an important role in understanding perception in relation to cognitive and ecological adaptation in a range of species.

\section{REFERENCES}

Blough, D. S. (1984). Form recognition in pigeons. In H. L. Roitblat, T. G. Bever, \& H. S. Terrace (Eds.), Animal cognition (pp. 277-289). Hillsdale, NJ: Erlbaum.

Cerella, J. (1980). The pigeon's analysis of picture. Pattern Recognition, 12, 1-6.

Cerella, J. (1990). Shape constancy in the pigeon: The perspective transformations decomposed. In M. L. Commons, R. J. Herrnstein, S. M. Kosslyn, \& D. B. Mumford (Eds.), Quantitative analyses of behavior (Vol. 8., pp. 145-163). Hillsdale, NJ: Erlbaum.

Cook, R. G., \& KaTz, J. S. (1999). Dynamic object perception by pigeons. Journal of Experimental Psychology: Animal Behavior Processes, 25, 194-210.

Dumas, C., \& WiLkie, D. M. (1995). Object permanence in ring doves (Streptopelia risoria). Journal of Comparative Psychology, 109, 142150.

Forkman, B. (1998). Hens use occlusion to judge depth in a twodimensional picture. Perception, 27, 861-867.

Fujita, K. (1996). Linear perspective and the Ponzo illusion: A comparison between rhesus monkeys and humans. Japanese Psychological Research, 38, 136-145.

FujITA, K. (1997). Perception of the Ponzo illusion by rhesus monkeys, chimpanzees, and humans: Similarity and difference in the three primate species. Perception \& Psychophysics, 59, 284-292.

Fujita, K., Blough, D. S., \& Blough, P. M. (1991). Pigeons see the Ponzo illusion. Animal Learning \& Behavior, 19, 283-293.

Fujita, K., Blough, D. S., \& Blough, P. M. (1993). Effects of the inclination of context lines on perception of the Ponzo illusion by pigeons. Animal Learning \& Behavior, 21, 29-34.

FUNK, M. S. (1996). Development of object permanence in the New Zealand parakeet (Cyanoramphus auriceps). Animal Learning \& Behavior, 24, 375-383.

Granrud, C. E., \& Yonas, A. (1984). Infants' perception of pictorially specified interposition. Journal of Experimental Child Psychology, 37, 500-511.

Grossberg, S., \& Mingolla, E. (1985). Neural dynamics of form perception: Boundary completion, illusory figures, and neon color spreading. Psychological Review, 92, 173-211.

Gunderson, V. M., Yonas, A., Sargent, P. L., \& Grant-Webster, K. S. (1993). Infant macaque monkeys respond to pictorial depth. Psychological Science, 4, 93-98.

Hodos, W. (1993). The visual capabilities of birds. In H. P. Zeigler \& 
H.-J. Bischof (Eds.), Vision, brain, and behavior in birds (pp. 63-76). Cambridge, MA: MIT Press.

Kanizsa, G. (1979). Organization in vision: Essays on Gestalt perception. New York: Praeger.

Kanizsa, G., Renzi, P., Conte, S., Compostela, C., \& Guerani, L. (1993). Amodal completion in mouse vision. Perception, 22, 713721.

Kellman, P. J., \& Spelke, E. S. (1983). Perception of partly occluded objects in infancy. Cognitive Psychology, 15, 483-524.

Lea, S. E. G., Slater, A. M., \& Ryan, C. M. E. (1996). Perception of object unity in chicks: A comparison with the human infant. Infant Behavior \& Development, 19, 501-504.

Pepperberg, I. M., \& Funk, M. S. (1990). Object permanence in four species of psittacine birds: An African Grey parrot (Psittacus erithacus), an Illiger mini macaw (Ara maracana), a parakeet (Melopsittacus undulatus), and a cockatiel (Nymphicus hollandicus). Animal Learning \& Behavior, 18, 97-108.

Plowright, C. M. S., ReId, S., \& Kilian, T. (1998). Finding hidden food: Behavior on visible displacement tasks by mynahs (Gracula religiosa) and pigeons (Columba livia). Journal of Comparative Psychology, 112, 13-25.

Regolin, L., \& Vallortigara, G. (1995). Perception of partly occluded objects by young chicks. Perception \& Psychophysics, 57, 971-976.
Sato, A., Kanazawa, S., \& Fujita, K. (1997). Perception of object unity in a chimpanzee (Pan troglodytes). Japanese Psychological Research, 39, 191-199.

Sekuler, A. B., Lee, J. A. J., \& Shettleworth, S. J. (1996). Pigeons do not complete partly occluded figures. Perception, 25, 1109-1120.

Sekuler, A. B., \& Palmer, S. E. (1992). Perception of partly occluded objects: A microgenetic analysis. Journal of Experimental Psychology: General, 121, 95-111.

Yin, C., Kellman, P. J., \& Shipley, T. F. (1997). Surface completion complements boundary formation in the visual integration of partly occluded objects. Perception, 26, 1459-1479.

YonAs, A., \& ARTERberry, M. E. (1994). Infants perceive spatial structure specified by line junctions. Perception, 23, 1427-1435.

\section{NOTE}

1. This criterion was employed because pigeons generally perform less accurately than primates.

(Manuscript received December 14, 1998; revision accepted for publication April 20, 2000.) 